\title{
Effect of dexmedetomidine on erythrocyte deformability during ischaemia-reperfusion injury of heart in diabetic rats
}

\author{
Arslan $\mathrm{M}^{1}$, Comu FM², Kip $\mathrm{G}^{1}$, Alkan $\mathrm{M}^{1}$, Kiraz $\mathrm{HA}^{3}$, Ozer A ${ }^{4}$, Sivgin $\mathrm{V}^{1}$ \\ Department of Anaesthesiology and Reanimation, Gazi University Medical Faculty, Ankara, Turkey. \\ marslan36@yahoo.com
}

\begin{abstract}
The aim of this study is to evaluate the effect of dexmedetomidine on erythrocyte deformability during IR heart injury in diabetic rats.

Methods: Eighteen Wistar Albino rats were included in the study after streptozocin $(55 \mathrm{mg} / \mathrm{kg})$ treatment for four weeks. In the Group C and DC (sham-control group), the coronary artery was not occluded or reperfused in the control rats. In the Group DIR, a branch of the left coronary artery was occluded for 30 minutes followed by two hours of reperfusion to produce IR. In the Group DIRD, a branch of the left coronary artery was occluded for 30 minutes followed by two hours of reperfusion to produce IR, and dexmedetomidine was administrated via 100 $\mu \mathrm{g} / \mathrm{kg}$ IP route 30 minutes before ligating the left coronary artery. Deformability measurements were performed in erythrocyte suspensions containing $\mathrm{Htc} 5 \%$ in a PBS buffer.

Results: The deformability index was significantly increased in diabetic rats; however, it was similar in the Group DC and DIRD. It was significantly increased in the Group DIR when compared to the Group C, DIRD and DC. The relative resistance was increased in IR models.

Conclusion: Erythrocyte deformability was decreased in rats having diabetes and IR injury. This injury might lead to further problems in microcirculation. It was shown that dexmedetomidine might be useful in enhancing the adverse effects of this type of injury (Fig. 1, Ref. 39). Text in PDF www.elis.sk.

Key words: erythrocyte deformability, myocardial ischemia reperfusion, experimental diabetes, dexmedetomidine, rat.
\end{abstract}

In last two or three decades, the prevalence of diabetes mellitus (DM) has rapidly increased throughout the world, and experts estimate that it will increase by $200 \%$ in the next several decades (1-4). Diabetes mellitus affects patients of all ages and, due to its increasing prevalence, most physicians will be confronted with diabetic patients requiring anaesthesia and surgery. Patients with diabetes have a significantly increased risk of premature mortality and an increased risk of microvascular and cardiovascular complications. These patients are often sicker than most non-diabetic patients; therefore, they place a proportionally larger burden on anaesthetic services $(2,5,6)$. Thus, more careful perioperative care is necessary for these patients (7).

A $50 \%$ increase in early mortality following coronary artery bypass grafting has been described in diabetic patients (5). One study demonstrated that the rate of unrecognised myocardial infarction was $39 \%$ in diabetic patients and $22 \%$ in non-diabetic patients (8). Cardiac surgery with cardiopulmonary bypass inevitably causes a systemic inflammatory response and ischemia-reperfusion (IR) injury affecting multiple organs $(1,2)$.

${ }^{1}$ Department of Anaesthesiology and Reanimation, Gazi University Medical Faculty, Ankara, Turkey, ${ }^{2}$ Department of Physiology, Kirikkale University Medical Faculty, Kirikkale, Turkey, ${ }^{3}$ Department of Anaesthesiology and Reanimation, Onsekiz Mart University Medical Faculty, Canakkale, Turkey, and ${ }^{4}$ Department of Cardiovascular Surgery, Gazi University Medical Faculty, Ankara, Turkey

Address for correspondence: M. Arslan, Dr, Gazi University Medical Faculty, Department of Anesthesiology and Reanimation, 06510 Ankara, Turkey. Phone: +90.312 .2026739$
Many tissues and cells can be damaged by free radicals, with red blood cells (RBC) being one of the most susceptible. During IR, the increased oxidative stress (OS) can cause augmented RBC membrane lipid peroxidation with the consequent alteration of cellular deformability. Erythrocyte deformability is of crucial importance for the maintenance of normal circulation, as it facilitates the passage of RBC through narrow capillaries in the microcirculation and reduces blood viscosity at high shear rates in large blood vessels (9).

Haemorheological parameters that include (but are not limited to) hematocrit, plasma proteins, erythrocyte aggregation and erythrocyte deformability in DM are often disturbed (10).

Several drugs in anaesthesia have been used to prevent IR injury, including sevoflurane, dexmedetomidine, isoflurane and ketamine (11-15).

Dexmedetomidine, a selective and potent $\alpha 2$ - adrenoceptor agonist, was approved by the US Food and Drug Administration (FDA) in 1999 for the sedation of patients hospitalized in intensive care settings. Since then, a growing number of research articles have emerged reporting other possible indications, such as regional and general anaesthesia $(16,17)$. Dexmedetomidine was reported to be effective in protecting against focal ischaemia in rabbits, in cardiac IR injury in rats, in kidney IR injury in rats and in incomplete forebrain ischaemia in rats $(12,18-20)$. Despite its increased clinical use in critically ill patients, the effects of dexmedetomidine on myocardial injury induced by left coronary artery (LAD) IR have not yet been investigated.

The primary aim of this study was to investigate deformability changes and the preventive role of dexmedetomidine against these 
changes in erythrocytes of diabetic rats during an experimental model of myocardial IR injury.

\section{Materials and methods}

\section{Animals and Experimental Protocol}

This study was conducted in the GUDAM Laboratory of Gazi University with the consent of the Experimental Animals Ethics Committee of Gazi University. All of the procedures were performed according to the accepted standards of the Guide for the Care and Use of Laboratory Animals.

In the study, 24 male Wistar Albino rats weighing between 200 and $250 \mathrm{~g}$, raised under the same environmental conditions, were used. The rats were kept at $20-21{ }^{\circ} \mathrm{C}$ in cycles of 12 hours of daylight and 12 hours of darkness and had free access to food until two hours before the anaesthetic procedure. The animals were randomly separated into the four groups, each containing six rats.

Diabetes was induced by a single IP injection of streptozotocin (Sigma Chemical, St. Louis, MO, USA), at a dose of $55 \mathrm{mg} . \mathrm{kg}^{-1}$ body weight. The blood glucose levels were measured 72 hours following this injection. Rats were classified as diabetic if their fasting blood glucose (FBG) levels exceeded $250 \mathrm{mg}^{-\mathrm{dl}^{-1}}$, and only animals with FBGs of $>250 \mathrm{mg}$.dl ${ }^{-1}$ were included in the diabetic groups (diabetes only, diabetes plus ischaemia-reperfusion and diabetes plus dexmedetomidine-ischaemia-reperfusion). The rats were kept alive for four weeks after streptozotocin injection to allow the development of chronic diabetes before they were exposed to ischaemia-reperfusion (21). The rats were weighed before the study.

Rats were anesthetized with an IP injection of $100 \mathrm{mg} \cdot \mathrm{kg}^{-1}$ of ketamine. The trachea was cannulated for artificial respiration. The chest was shaved and each animal was fixed in a supine position on the operating table. The chest was opened by a left thoracotomy followed by sectioning the fourth and fifth ribs about $2 \mathrm{~mm}$ to the left of the sternum. Positive-pressure artificial respiration was started immediately with room air, using a volume of $1.5 \mathrm{ml} / 100$ $\mathrm{g}$ body weight at a rate of $60 \mathrm{strokes} / \mathrm{min}$. Sodium heparin (500 $\mathrm{IU} / \mathrm{kg}$ ) was administered through the peripheral vein in the tail.

After the pericardium was incised, the heart was exteriorized with gentle pressure on the right side of the rib cage. An 8/0 silk suture attached to a 10-mm micropoint reverse-cutting needle was quickly placed under the left main coronary artery. The heart was then carefully replaced in the chest and the animal was allowed to recover for 20 minutes.

There were four experimental groups. The Group C (control; $\mathrm{n}=6$ ), Group DC (diabetes-control; $\mathrm{n}=6$ ), Group DIR (diabetes-ischaemia-reperfusion; $\mathrm{n}=6$ ) and Group DIRD (diabetesischaemia-reperfusion-dexmedetomidine; $\mathrm{n}=6$ ) underwent left thoracotomy and received IP dexmedetomidine (Precedex 100 $\mu \mathrm{g} / 2 \mathrm{ml}$, Abbott $^{\circledR}$, Abbott Laboratory, North Chicago, IL, USA) administrated via $100 \mathrm{mg} \cdot \mathrm{kg}^{-1} \mathrm{IP}$ route 30 minutes before ligating the LAD (13). A small plastic snare was threaded through the ligature and placed in contact with the heart. The artery could then be occluded by applying tension to the ligature (30 minutes), and reperfusion was achieved by releasing the tension (120 minutes) (12). However, after the above procedure, the coronary artery was not occluded or reperfused in the control and diabetic control rats.

All the rats were given ketamine $100 \mathrm{mg} \cdot \mathrm{kg}^{-1} \mathrm{IP}$ and intracardiac blood samples were obtained. Heparinized total blood samples were used to prepare erythrocyte packs. Deformability measurements were performed using erythrocyte suspensions with $5 \%$ haematocrit in a phosphate buffered saline (PBS) buffer.

\section{Deformability measurements}

Blood samples were carefully taken and the measurement process was as fast as possible to avoid haemolysis of erythrocytes. The collected blood was centrifuged at $1000 \mathrm{rpm}$ for ten minutes. Serum and buffy coat on erythrocytes were removed. An isotonic PBS buffer was added to the collapsing erythrocytes and this was centrifuged at $1000 \mathrm{rpm}$ for ten minutes. Liquid on the upper surface was removed. Finally, pure red cell packs were obtained from the washing process, which was repeated three times. Erythrocyte packs were mixed with the PBS buffer to generate a suspension with a value of $5 \% \mathrm{Htc}$. These erythrocyte suspensions were used for the measurement of deformability. Collection and deformability measurements of erythrocytes were performed at $22{ }^{\circ} \mathrm{C}$.

The constant-current filtrometer system was used in the measurement of erythrocyte deformability. Samples to be measured were prepared with $10 \mathrm{ml}$ of erythrocyte suspension and PBS buffer. The flow rate was held constant at $1.5 \mathrm{ml} / \mathrm{min}$ with an infusion pump. A $28 \mathrm{~mm}$ nucleoporin polycarbonate filter with a $5 \mu \mathrm{m}$ pore diameter was preferred. Pressure changes while the erythrocytes passed through the filter were detected by the pressure transducer, and the data was transferred to the computer with the help of an MP30 data equation system (Biopac Systems Inc, Commat, USA). The necessary calculations were performed with related computer programs by measuring the pressure changes at various times. Pressure calibration of the system was performed before each sample measurement. The buffer $\left(\mathrm{P}_{\mathrm{T}}\right)$ and the erythrocytes $\left(\mathrm{P}_{\mathrm{E}}\right)$ were passed through the filtration system and the changes in pressure were measured. The relative refractory period value (Rrel) was calculated by relating the pressure value of the erythrocyte suspension to the pressure value of buffer. Increasing

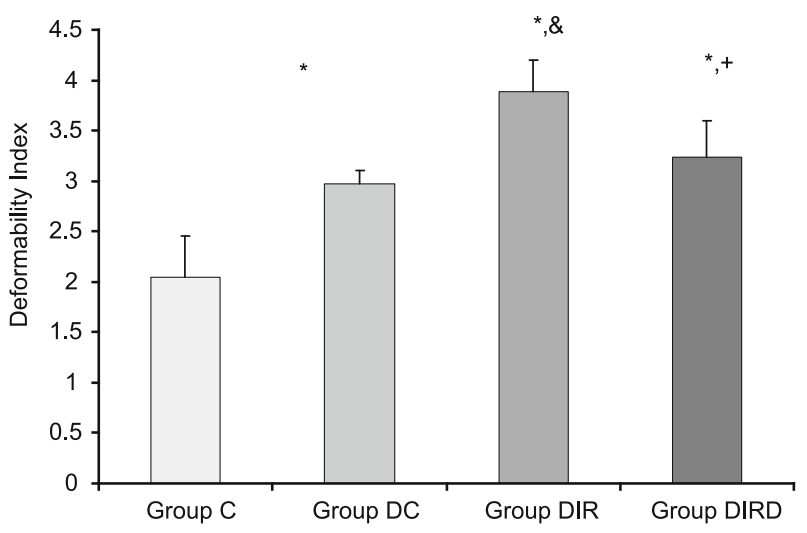

Fig. 1. Erythrocyte deformability values of the groups. Each bar represents the mean \pm SD. $*$ p $<0.05$ compared to the Group C; \& p $<0.05$ compared to the Group DC; + $\mathbf{p}<0.05$ compared to the Group DIR. 
Rrel in the deformability index was interpreted to adversely affect the erythrocytes' deformability $(22,23)$.

\section{Statistical analysis}

The Statistical Package for the Social Sciences (SPSS, Chicago, IL, USA) 12.0 program was used for the statistical analysis. Variations in blood glucose levels, erythrocyte deformability and rat weights between study groups were assessed using the KruskalWallis test. The Bonferroni-adjusted Mann-Whitney U test was used after significant Kruskal-Wallis to determine which groups differed from the others. Results were expressed as the mean \pm standard deviation (Mean \pm SD). Statistical significance was set at the $p$ value of $<0.05$ for all analysis and $p<0.033(0.1 / 3)$ for Bonferroni-adjusted Mann-Whitney U.

\section{Results}

Blood glucose measurements were $85.8 \pm 10.4,335.4 \pm 50.5$, $343.7 \pm 66.3$ and $338.3 \pm 55.8 \mathrm{mg} / \mathrm{dL}$ for Group C, DC, DIR and DIRD, respectively. Serum glucose was detected to be significantly lower in the Group C when compared to the Groups DC, DIR and $\operatorname{DIRD}(\mathrm{p}<0.0001)$.

The deformability index was significantly increased in the diabetic rats $(\mathrm{p}<0.0001)$; however, it was similar in the Group DC and DIRD $(p=0.912)$. It was significantly increased in the Group DIR when compared to the Group C, DC and DIRD ( $p<0.0001$, $\mathrm{p}<0.0001, \mathrm{p}=0.011$, respectively) (Fig. 1). Relative resistance was increased in IR models.

\section{Discussion}

Diabetes mellitus (DM) is a metabolic disorder characterized by abnormally high blood sugar (hyperglycaemia) resulting from either low insulin levels or insulin resistance in most of the body cells. Diabetes mellitus has a high social and economic importance, as the number of diabetes patients continues to grow at an unprecedented rate throughout the world (10).

Haemorheological parameters, such as haematocrit, plasma proteins, erythrocyte aggregation and erythrocyte deformability are often disturbed in DM (24). For migration of oxygen and vital molecules to the final organ capillaries and clearance of metabolic wastes, erythrocytes must be able to extend and curve and have the capability to move in these areas. This capacity, called "deformability", becomes more important in microcirculation. Altered erythrocyte deformability not only changes the oxygen delivery capacity of the erythrocytes but also the survival of the circulating erythrocytes (25-27).

Additionally, it has been suggested that the impaired perfusion at the tissue level observed as a complication of DM is primarily due to reduced erythrocyte deformability $(28,29)$. In addition, metabolic changes and tissue perfusion due to cardiovascular problems may lead to an inadequate recovery in plasma viscosity (30).

Cho et al (10) demonstrated that blood viscosity significantly increased in diabetes. These results suggest that the consequent elevation of glucose in blood plasma primarily affects RBCs and the vascular endothelial cells, including the walls of capillaries. The impaired glucose tolerance or uncontrolled blood glucose levels often result in microvascular complications in diabetes. Moreover, the impairment of erythrocyte deformability is attributed to the specific changes in the membrane structure. The oxidative stress due to high glucose concentrations causes damage to the erythrocyte membrane proteins, even with a relatively short exposure time (31).

Barnes et al (32) showed that erythrocyte deformability was lower in 14 diabetes patients with the most extensive micro-angiopathy than in the controls or 22 diabetes patients with slight or no complications. They suggested that hyperviscosity and reduced erythrocyte deformability may be important and potentially treatable factors in the aetiology or progression of microcirculatory disease in diabetes. Similar to these previous studies, we also found that erythrocyte deformability was decreased in rats with induced diabetes.

In this study, for the first time to our knowledge, we reported that IR of the diabetic rat heart resulted in significant negative changes that can be observed in erythrocyte deformability and that dexmedetomidine, a highly potent and selective $\alpha 2$-adrenoreceptor agonist, administered at the beginning of heart ischaemia, can provide varying degrees of protection against negative effects of variations in erythrocyte deformability.

Dexmedetomidine is a highly selective alpha-2 agonist that provides sedation and analgesia without respiratory depression. It activates pro-survival kinases and attenuates ischaemia and hypoxic injury, including cardioprotection (33), neuroprotection (34) and renoprotection (35). Concurrent infusion during surgery reduces anaesthetic consumption by $20-50 \%$ (36) and produces a moderate decrease in heart rate and blood pressure that may be advantageous in ischaemic heart disease by improving the balance of oxygen supply and demand (37).

In a study regarding $\alpha 2$ agonists, Belhoula et al (38) reported that premedication of type- 2 diabetic patients with clonidine 90 minutes before surgery improved blood glucose control and decreased insulin requirements during ophthalmic surgery. The clonidine decreased circulating catecholamines despite having no effect on cortisol concentrations and GH secretion. However, Venn et al (39) reported that dexmedetomidine decreased insulin secretion after major surgery without exacerbating the glycaemic response. This report suggested that an impaired insulin secretion was balanced by reduced sympathetic activity. At present, it would be reasonable to accept that $\alpha 2$ agonists, such as clonidine or dexmedetomidine, modify insulin secretion without exacerbating the glycaemic response.

In conclusion, the results of this study clearly demonstrated that erythrocyte deformability is significantly altered in the experimental myocardial IR injury in the diabetic rat. This might lead to further problems in microcirculation. Thus, measurement of erythrocyte deformability might have an important impact on the follow-up for IR injury. Additionally, dexmedetomidine administered before induction of ischaemia was observed to have protective effects on these alterations in myocardial IR injury. Other aspects of these findings, including clinical significance and practical applications, merit further experimental and clinical investigation. 


\section{References}

1. Robertshaw HJ, Hall GM. Diabetes mellitus: anaesthetic management. Anaesthesia 2006; 61: 1187-1190.

2. McAnulty GR, Robertshaw HJ, Hall GM. Anaesthetic management of patients with diabetes mellitus. Br J Anesth 2000; 85: 80-90.

3. McAnulty GR, Hall GM. Anaesthesia for the diabetic patient. Br J Anesth 2003; 88: 428-430.

4. Gu W, Pagel PS, Warltier DC, Kersten JR. Modifying cardiovascular risks in diabetes mellitus. Anesthesiology 2003; 98: 774-779.

5. Giquel J, Rodriguez-Blanco YF, Matadial C, Candiotti K. Diabetes mellitus in anaesthesia. Br J Diabetes Vasc Dis 2012; 12: 60-64.

6. Miller RD. Miller's Anesthesia. 7th ed. In: Roizen MF, Fleisher LA, eds. Anesthesia implications of current disease. Chicago: Year Book Medical Publishers, 2010: 1067-1149.

7. Kadoi Y. Anesthetic considerations in diabetic patients. Part I: preoperative considerations of patients with diabetes mellitus. J Anesth 2010; 24: 739-747.

8. Margolis JR, Kannel WS, Feinleib M, Dawber TR, McNamara PM. Clinical features of unrecognized myocardial infarction silent and symptomatic. Eighteen year follow-up: Framingham study. Am J Cardiol 1973; 32: 1-7.

9. Peto K, Nemeth N, Brath E, Takacs IE, Baskurt OK, Meiselman HJ et al. The effects of renal ischemia-reperfusion on hemorheological factors: preventive role of allopurinol. Clin Hemorheol Microcirc 2007; 37 (4): 347-358.

10. Cho YI, Mooney MP, Cho DJ. Hemorheological disorders in diabetes mellitus. J Diabetes Sci Technol 2008; 2 (6): 1130-1138.

11. Bedirli N, Ofluoglu E, Kerem M, Utebey G, Alper M, Yilmazer D et al. Hepatic energy metabolism and the differential protective effects of sevoflurane and isoflurane anesthesia in a rat hepatic ischemia-reperfusion injury model. Anesth Analg 2008; 106 (3): 830-837.

12. Kocoglu H, Karaaslan K, Gonca E, Bozdogan O, Gulcu N. Preconditioning effects of dexmedetomidine on myocardial ischemia/reperfusion injury in rats. Curr Ther Res Clin Exp 2008; 69: 150-158.

13. Arslan M, Comu FM, Isik B, Oztürk L, Kesimci E. Effect of dexmedetomidine on erythrocyte deformability during ischemia-reperfusion injury of liver in diabetic rats. Bratisl Med J 2012; 113 (12): 687-691.

14. Kim M, Kim M, Kim N, D’Agati VD, Emala CW, Lee HT. Isoflurane mediates protection from renal ischemia-reperfusion injury via sphingosine kinase and sphingosine-1-phosphate-dependent pathways. Am J Physiol Renal Physiol 2007; 293 (6): F1827-1835.

15. Guzmán-De La Garza FJ, Cámara-Lemarroy CR, Ballesteros-Elizondo RG, Alarcon-Galvan G, Cordero-Perez P, Fernandez-Garza NE. Ketamine reduces intestinal injury and inflammatory cell infiltration after ischemia/reperfusion in rats. Surg Today 2010; 40 (11): 1055-1062.

16. McCutcheon CA, Orme RM, Scott DA, Davies MJ, McGlade DP. A comparison of dexmedetomidine versus conventional therapy for sedation and hemodynamic control during carotid endarterectomy performed under regional anesthesia. Anesth Analg 2006; 102: 668-675.

17. Ramsay MA, Luterman DL. Dexmedetomidine as a total intravenous anesthetic agent. Anesthesiology 2004; 101: 787-790.

18. Maier CM, Sun GH, Kunis DM, Giffard RG, Steinberg GK. Neuroprotection by the N-methyl-D-aspartate receptor antagonist CGP 40116: In vivo and in vitro studies. J Neurochem 1995; 65: 652-659.

19. Kocoglu H, Ozturk H, Ozturk H, Yilmaz F, Gulcu N. Effect of dexmedetomidine on ischemia-reperfusion injury in rat kidney: a histopathologic study. Ren Fail 2009; 31 (1): 70-74.

20. Hoffman WE, Kochs E, Werner C, Thomas C, Albrecht RF. Dexmedetomidine improves neurologic outcome from incomplete ischemia in the rat. Reversal by the alpha 2-adrenergic antagonist atipamezole. Anesthesiology 1991; 75: 328-332.
21. Türeci E, IS M, Üzüm G, Akyüz F, Ulu MO, DöSoglu M et al. Alterations in blood-brain barrier after traumatic brain injury in streptozotocin-induced diabetic rats. J Nervous Sys Surgery 2009; 2 (2): 79-86.

22. Arslan M, Comu FM, Isik B, Unal Y, Cekmen N, Kurtipek O. Effects of a general anaesthetic agent, propofol, on erythrocyte deformability. Bratisl Med J 2010; 111 (3): 126-128.

23. Tatlican S, Duran FS, Eren C, Eskoglu F, Dikmenoglu N, Oktay B et al. Reduced erythrocyte deformability in active and untreated BehCet's disease patients. Int J Dermatol 2010; 49 (2): 167-171.

24. Barnes A, Willars E. Diabetes. In: Chien S, Dormandy J, Ernst E, A Matrai A(Eds). Clinical Hemorheology. Dordrecht: Martinus Nijhoff, 1987: 275-309.

25. Zinchuk VV. Erythrocyte deformability: physiological aspects. Usp Fiziol Nauk 2001; 32 (3): 66-78.

26. KuypersFA. Red cell membrane damage. J Heart Valve Dis 1998; 7: 387-395.

27. Sivilotti ML. Oxidant stress and haemolysis of the human erythrocyte. Toxicol Rev 2004; 23: 169-188.

28. Le Devehat C, Khodabandehlou T, Vimeux M. Relationship between hemorheological and microcirculatory abnormalities in diabetes mellitus. Diabet Metab 1994; 20 (4): 401-404.

29. Zimny S, Dessel F, Ehren M, Pfohl M, Schatz H. Early detection of microcirculatory impairment in diabetic patients with foot at risk. Diabet Care 2001; 24 (10): 1810-1814.

30. Muller R, Musikic P. Hemorheology in surgery: a review. Angiology 1987; 38 (8): 581-592.

31. Mataseje A, Beder I, Kittova M, Okkelova J, Vazan R. The assessment of erythrocyte deformability by filtration rate. Bratisl Med J 2003; 104 (4-5): 158-160.

32. Barnes AJ, Locke P, Scudder PR, Dormandy TL, Dormandy JA, Slack J. Is hyperviscosity a treatable component of diabetic microcirculatory disease? Lancet 1977; 2 (8042): 789-791.

33. Ibacache M, Sanchez G, Pedrozo Z, Galvez F, Humeres C, Echevarria $\mathbf{G}$ et al. Dexmedetomidine preconditioning activates pro-survival kinases and attenuates regional ischemia/reperfusion injury in rat heart. Biochim Biophys Acta 2012; 1822: 537-545.

34. Zhu YM, Wang CC, Chen L, Qian LB, Ma LL, Yu J et al. Both PI3K/ Akt and ERK1/2 pathways participate in the protection by dexmedetomidine against transient focal cerebral ischemia/reperfusion injury in rats. Brain Res 2013; 1494: 1-8.

35. Gu J, Sun P, Zhao H, Watts HR, Sanders RD, Terrando N et al. Dexmedetomidine provides renoprotection against ischemia-reperfusion injury in mice. Crit Care 2011; 15: R153.

36. Aantaa R, Jaakola ML, Kallio A, Kanto J. Reduction of the minimum alveolar concentration of isoflurane by dexmedetomidine. Anesthesiology 1997; 86: 1055-1060.

37. Biccard BM, Goga S, de Beurs J. Dexmedetomidine and cardiac protection for non-cardiac surgery: a meta-analysis of randomised controlled trials. Anaesthesia 2008; 63: 4-14.

38. Belhoula M, Ciebiera JP, De La Chapelle A, Boisseau N, Coeurveille D, Raucoules-Aime M. Clonidine premedication improves metabolic control in type 2 diabetic patients during ophthalmic surgery. Br J Anesth 2003; 90: 434-439.

39. Venn RM, Bryant A, Hall GM, Grounds RM. Effects of dexmedetomidine on adrenocortical function, and the cardiovascular, endocrine and inflammatory responses in postoperative patients needing sedation in the intensive care unit. Br J Anesth 2001; 86: 650-656.

Received August 18, 2013. Accepted April 23, 2014. 